\title{
A prospective phase I study of hypo- fractionated neoadjuvant radiotherapy for locally advanced gastric cancer
}

Ning Li ${ }^{1}$, Xin Wang ${ }^{1}$, Yuan Tang ${ }^{1}$, Dongbing Zhao ${ }^{2}$, Yihebali Chi ${ }^{3}$, Lin Yang ${ }^{3}$, Liming Jiang ${ }^{4}$, Jun Jiang ${ }^{4}$, Wenyang Liu', Yu Tang ${ }^{1}$, Hui Fang ${ }^{1}$, Yueping Liu', Yongwen Song ${ }^{1}$, Shulian Wang ${ }^{1}$, Jing Jin ${ }^{1 *}$ and Yexiong Li ${ }^{1}$

\begin{abstract}
Background: Previous studies have reported that neoadjuvant chemoradiotherapy can downstage the advanced gastric cancer. However, no studies are available on the application of hypo-radiotherapy to neoadjuvant radiotherapy. This study sought to assess the maximum tolerated dose (MTD) and dose-limited toxicity (DLT) of hypo-fractionated chemoradiotherapy for local advanced gastric cancer.

Method: Patients with cT3-4 and/or lymph node-positive locally advanced gastric cancer or Siewert II/III esophagogastric junction adenocarcinoma were enrolled. Preoperative chemoradiation was followed by 3 cycles of oxaliplatin + S-1 neoadjuvant chemotherapy with an interval duration of 3-4 weeks. D2 resection was performed 2-4 weeks after neoadjuvant therapy. Three cycles of adjuvant chemotherapy were planned after surgery. Intensitymodulated radiotherapy (IMRT) was used. The radiotherapy dose level was defined using three levels, namely, 40. 0 Gy/2.5 Gy, 41.6 Gy/2.6 Gy, 43.2 Gy/2.7 Gy delivered concurrently with S-1 at 80 mg/m².
\end{abstract}

Results: From May 2016 to Dec 2016, nine patients with a median age of 63 years were enrolled in this study. The most common grade I-III adverse events were leukopenia (88.9\%), nausea (88.9\%), vomiting (77.8\%) and weight loss (66.7\%). Grade III adverse events consisted of vomiting and weight loss.

Conclusion: The MTD of hypo-fractionated radiotherapy for locally advanced gastric cancer was 40.0 Gy/2.5 Gy, and the DLTs were vomiting and weight loss.

Trial registration: Clinicaltrials.gov ID: NCT03427684 (Retrospectively registered on February 9, 2018).

Keywords: Gastric cancer, Chemoradiotherapy, Phase I study, Hypo-fractionated radiotherapy

\section{Background}

Gastric cancer is one of the most common malignant tumors in China, and the incidence and mortality rates for this disease rank second among all malignant tumors [1]. Gastric cancer requires integrated multidisciplinary treatment, and surgery is currently the only possible curative method. Previous studies have reported that neoadjuvant chemoradiotherapy can downstage the primary tumor to increase the radical resection rate for improved long-term prognosis of advanced gastric cancer

\footnotetext{
* Correspondence: jinjing@csco.org.cn

1Department of Radiation Oncology, Chinese National Cancer Center/Cancer Hospital, Chinese Academy of Medical Sciences, 17 Panjiayuannanli, Chaoyang District, Beijing 100021, China

Full list of author information is available at the end of the article
}

[2-5]. However, no studies are available on the application of hypo-radiotherapy to neoadjuvant radiotherapy in gastric cancer. The aim of this study was to observe the maximum tolerated dose (MTD) and dose-limited toxicity (DLT) of hypo-fractionated radiotherapy for locally advanced gastric cancer.

\section{Methods}

\section{Inclusion criteria}

Clinical stage T3-4 N + M0 gastric cancer or Siewert II/ III esophagogastric junction carcinoma; pathologically confirmed adenocarcinoma; $18-75$ years old, male or female; Karnofsky score $\geq 70$; white blood cell count $\geq 4 \times$ $10^{9} / \mathrm{L}$; platelet count $\geq 100 \times 10^{9} / \mathrm{L}$; serum creatinine $\leq 1 \times$ upper limit of normal, total bilirubin $\leq 1 \times$ upper limit of

(c) The Author(s). 2018 Open Access This article is distributed under the terms of the Creative Commons Attribution 4.0 International License (http://creativecommons.org/licenses/by/4.0/), which permits unrestricted use, distribution, and 
normal, alanine aminotransferase and aspartate aminotransferase $\leq 2.5 \times$ upper limit of normal, and alkaline phosphatase $\leq 5 \times$ upper limit of normal.

\section{Treatment procedure}

After enrollment in the study, patients were first treated with radiotherapy concurrent with oral S-1 at $80 \mathrm{mg} / \mathrm{m}^{2}$ / day on radiotherapy days. Three weeks after the end of radiotherapy, patients were treated with neoadjuvant chemotherapy with oxaliplatin and S-1 (SOX) for 3 cycles. Oxaliplatin was given at a dose of $130 \mathrm{mg} / \mathrm{m}^{2}$ intravenous (iv) on day 1 , and S-1 at 40-60 mg orally BID was given on days 1-14. Imaging evaluation was performed 3 weeks after neoadjuvant treatment (Fig. 1). The radical operation and surgical procedures were determined based on multidisciplinary team (MDT) discussion. Non-operable patients continued with 3 cycles of chemotherapy, and the chemotherapy regimen could be changed. Three cycles of SOX adjuvant chemotherapy were performed after surgery.

\section{Radiotherapy and dose escalation method}

The patients were fasting for more than $4 \mathrm{~h}$ before positioning, and a CT scan was performed after body film fixation. According to gastroscopy, MRI and CT, we determined the GTV range of primary tumors and lymph nodes. CTV includes GTV with $2.5 \mathrm{~cm}$ expanded in the mucosal direction and GTVnd. According to the location of the primary tumor, CTV included elective lymph nodes (LNs) regions (Table 1). Peri-gastric lymph node regions without GTVnd were excluded from the CTV. PTV was based on $7 \mathrm{~mm}$ radial and $10 \mathrm{~mm}$ proximal and distal expansions from CTV. The whole lung, liver, kidney, heart and spinal cord were contoured as normal tissue. The plan met the dose constraints to critical organs. The maximum dose of spinal cord could not exceed $45 \mathrm{~Gy}$; lung V $20<20 \%$, V 5 of the entire lung should have been less than 2300 CC; kidney V $20<30 \%$; cardiac V $40<30 \%$, V $25<$ $50 \%$; liver V30 < 30\%; small intestine V15 $<275$ CC, V40 < 150 CC. Intensity-modulated radiotherapy (IMRT), or volumetric-modulated arc radiotherapy (VMAT) technique was used (Fig. 2). We used the image-guided radiotherapy at the same time to ensure the accuracy of the position.

DLT was defined as the presence of the following adverse events during radiotherapy and within 1 month after
Table 1 Post-operative radiation elective lymph node regions

\begin{tabular}{|c|c|}
\hline Primary tumor segment & Elective lymph nodes regions \\
\hline Siewert II/III or proximal gastric cancer & $7,8,9,11 p, 16 a 2,16 b 1^{b}$ \\
\hline Middle or distal gastric cancer & $7,8,9,11 p, 12 a, 13,14^{a}, 16 a 2,16 b 1^{b}$ \\
\hline
\end{tabular}

the end of radiotherapy: $\geq$ grade 4 myelosuppression (leukopenia or thrombocytopenia), $\geq$ grade 3 non-blood adverse events (such as nausea, vomiting, fatigue, fever, pain, weight loss and other symptoms associated with treatment), and $\geq$ grade 2 abnormal renal and liver function. The dose of radiotherapy was increased progressively as follows: reference dose $40 \mathrm{~Gy} / 2.5 \mathrm{~Gy} / 16$ fractions, $95 \%$ isodose line covering the planning target volume (PTV); 95\% PTV 41.6 Gy/2.6 Gy/16 fractions; and 95\% PTV $43.2 \mathrm{~Gy} / 2.7 \mathrm{~Gy} / 16$ fractions. At least 3 patients were treated in each dose group. If DLT was not found in $3 \mathrm{pa}-$ tients, then the step to the next dose level was applied until the DLT occurred. If more than 2 DLTs occurred, the dose was the DLT dose level. If a dose group showed 1 case of DLT in the first 3 cases, the other 3 cases were added to the dose level. If the additional 3 cases did not incur DLT, the dose level was escalated to a higher level. If one or more DLTs appeared in the additional 3 cases, the dose level was the MTD level. If the above two circumstances appeared, the dose escalation was terminated, and the previous dose level was chosen as the MTD, which is also the recommended dose for a phase II clinical study.

\section{Preoperative assessment and postoperative pathological evaluation}

Preoperative TNM staging was evaluated via thoracic, abdominal and pelvic CT, gastroscopy, endoscopic ultrasonography and gastric MRI. PET scan and diagnostic laparoscopy were not mandatory. Surgical resection specimens were subjected to the Mandard tumor regression grade (TRG) classification standard to conduct an overall evaluation of primary lesions and lymph nodes. Grade 1 indicated complete regression; grade 2 showed fibrotic tissue with a small amount of residual cancer cells; grade 3 was assigned when cancer cells were present, but the

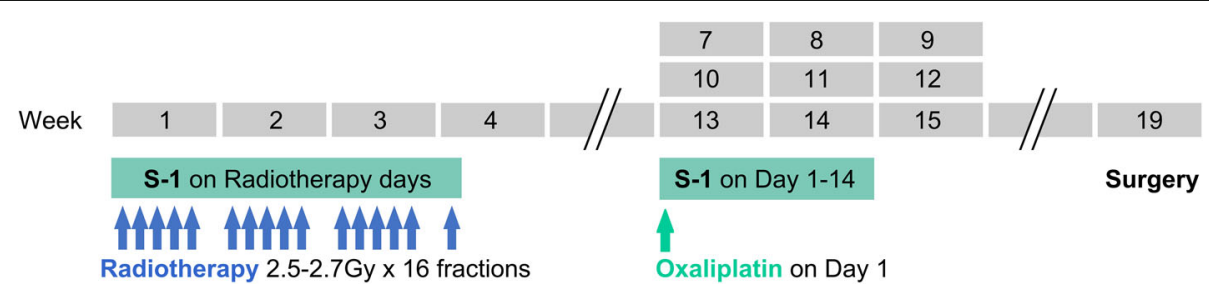

Fig. 1 Treatment schedule 


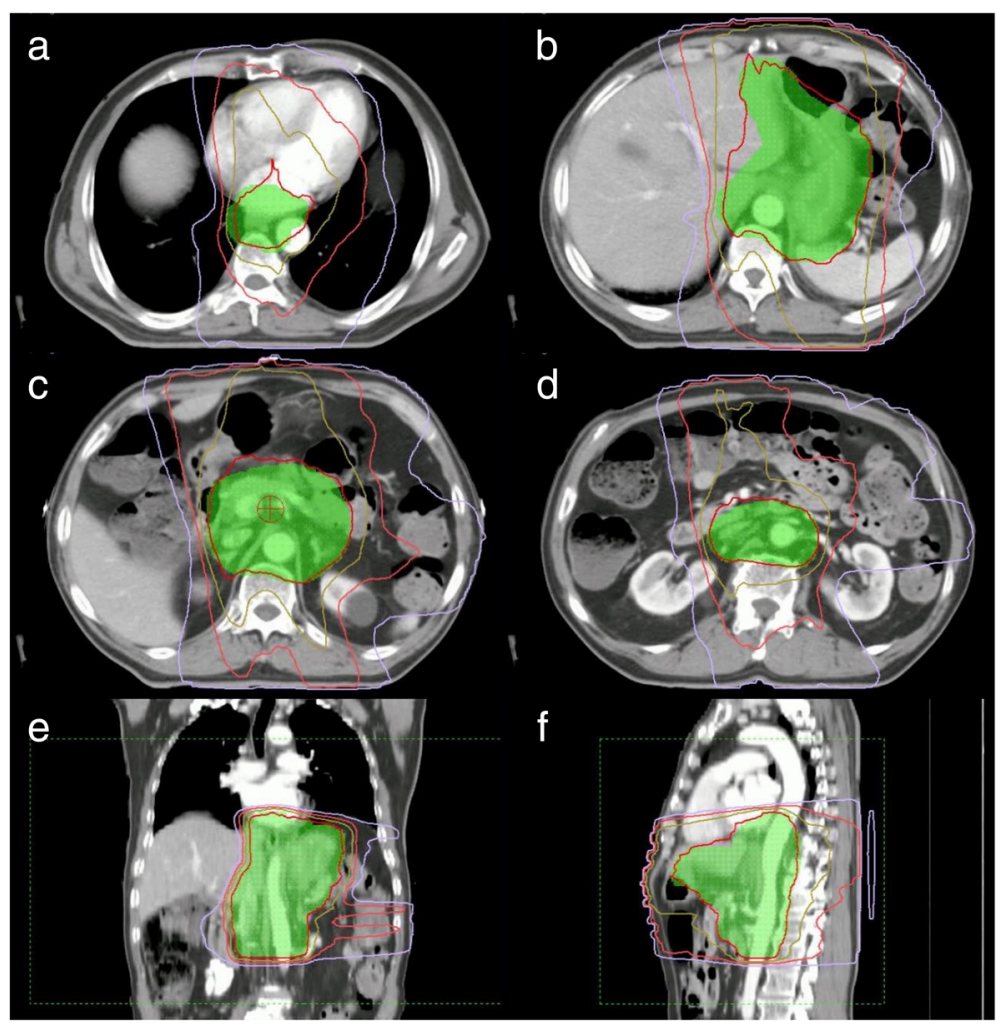

Fig. 2 Dose distributions of $250 \mathrm{cGy} \times 16$ fractions radiotherapy plan with 6MV-X based volumetric-modulated arc radiotherapy (VMAT) on a top level, b GTV level, c center level, d bottom level, e sagittal view and f coronal view. Isodose line of 1000 cGy, 2000 cGy, 3000 cGy, 4000 cGy and 4400 cGy were colored in purple, rose red, gold, red and yellow, respectively

residual fibrosis was dominant; grade 4 showed a greater proportion of residual cancer cells over fiber degeneration; and grade 5 indicated no signs of regression [6].

\section{Patient follow-up}

Follow-up occurred at 3-month intervals for 2 years, then at 6-month intervals until 5 years. Diagnostic evaluations were performed using $\mathrm{CT}$ of the chest and abdomen, and MRI or gastroscopy only if necessary.

\section{Statistical method}

The Kaplan-Meier method was used to calculate survival rate using SPSS 22.0 software (IBM, New Orchard Road Armonk, New York 10,504). The survival calculation was determined from the date of enrollment to death or to the last follow-up visit.

\section{Results}

\section{Patient characteristics}

From May 2016 to Dec 2016, 9 patients provided written consent. All the enrolled patients were male, and the median age was 63 years old (53-67). The Karnofsky scores of 8 cases were 90 , and 1 case had a score of 80 . There were 4 cases of Siewert II/III esophagogastric junction carcinoma and 5 cases of gastric cancer. There were 4 cases in stage IIIA, 2 in stage IIIB, 1 in stage IIIC and 2 in stage IIB (Table 2).

\section{Radiotherapy dose escalation results}

Of the 9 patients who completed the 2-level dose escalation trial, the $40 \mathrm{~Gy} / 2.5$ Gy group did not exhibit any DLTs. In the first 3 cases at the $41.6 \mathrm{~Gy} / 2.6$ Gy dose level, DLT occurred in 1 patient (grade 3 gastrointestinal toxicity), and 1 DLT occurred in an additional 3 cases (grade 3 weight loss) (Table 3). Therefore, $41.6 \mathrm{~Gy} / 2.6$ Gy was the DLT dose, and 40 Gy/2.5 Gy was the MTD dose.

\section{Side effects}

The most common grade 1-3 adverse events were leukopenia $(88.9 \%)$, nausea $(88.9 \%)$, vomiting $(77.8 \%)$ and weight loss (66.7\%). The toxicity at grade 3 consisted of vomiting and weight loss (Table 4). Of the 9 cases, one patient's concurrent chemoradiotherapy was interrupted for 7 days due to grade 3 vomiting.

\section{Completion of treatment}

All the patients completed concurrent chemoradiotherapy, and one patient refused any other treatments after 
Table 2 Patient characteristics

\begin{tabular}{ll}
\hline & Number (Percent) \\
\hline Median age (Range) & $63(53-67)$ \\
Gender & \\
Male & $9(100.0)$ \\
KPS score & \\
90 & $8(88.9)$ \\
80 & $1(11.1)$ \\
Segment & \\
Siewert II/II & $4(44.4)$ \\
Gastric & $5(55.6)$ \\
T stage & \\
T3 & $1(11.1)$ \\
T4a & $6(66.7)$ \\
T4b & $2(22.2)$ \\
N stage & \\
N0 & \\
N1 & $1(11.1)$ \\
N2 & $6(66.7)$ \\
Clinical stage & $2(22.2)$ \\
IIIA & \\
\hline III & $2(22.2)$ \\
& $4(44.4)$ \\
\hline & $2(22.2)$ \\
\hline & $1(11.1)$ \\
\hline
\end{tabular}

concurrent chemoradiotherapy. The other 8 patients received 3 cycles of neoadjuvant chemotherapy. The locoregional clinical response rate for the neo-adjuvant treatment was $100 \%$ (complete response rate $=22.2 \%$, partial response rate $=77.8 \%$ ). Four patients underwent radical surgery. Of the 5 patients who did not receive surgery, 1 patient refused the operation. The other 4 patients were unable to undergo the operation because of distant metastasis, and the alternative chemotherapy regimen was given for systemic therapy.

\section{Short-term efficacy and survival}

The postoperative pathological complete response (pCR) rate in the surgery group was $50 \%$, with pathological stage 0 in 2 cases, stage IIa in 1 case, and stage IIIa in 1 case. TRG grades 1, 2 and 3 consisted of 2 cases, 1 case

Table 3 Dose-limited toxicity

\begin{tabular}{llllll}
\hline $\begin{array}{l}\text { Dose } \\
\text { level }\end{array}$ & Dose & $n$ & $\begin{array}{l}\text { Number } \\
\text { of DLT }\end{array}$ & DLT & $\begin{array}{l}\text { Radiotherapy } \\
\text { dose when } \\
\text { DLT }\end{array}$ \\
\hline 1 & $40 \mathrm{~Gy} / 2.5 \mathrm{~Gy}$ & 3 & 0 & - & - \\
2 & $41.6 \mathrm{~Gy} / 2.6 \mathrm{~Gy}$ & 6 & 2 & $\begin{array}{l}\text { Vomiting } \\
\text { Weight loss }\end{array}$ & $\begin{array}{l}31.2 \mathrm{~Gy} \\
\end{array}$ \\
& & & & 41.6 Gy \\
\hline
\end{tabular}

Table 4 Toxicity incidence of hypo-fractionation radiotherapy for gastric cancer [n (\%)]

\begin{tabular}{llllll}
\hline & Grade 0 & Grade 1 & Grade 2 & Grade 3 & Grade 4 \\
\hline Leukopenia & $1(11.1)$ & $7(77.8)$ & $1(11.1)$ & $0(0)$ & $0(0)$ \\
Anemia & $8(88.9)$ & $1(11.1)$ & $2(22.2)$ & $0(0)$ & $0(0)$ \\
Thrombocytopenia & $1(11.1)$ & $1(11.1)$ & $2(22.2)$ & $0(0)$ & $0(0)$ \\
ALT & $9(100)$ & $0(0)$ & $0(0)$ & $0(0)$ & $0(0)$ \\
AST & $9(100)$ & $0(0)$ & $0(0)$ & $0(0)$ & $0(0)$ \\
TBIL & $9(100)$ & $0(0)$ & $0(0)$ & $0(0)$ & $0(0)$ \\
Nausea & $1(11.1)$ & $4(44.4)$ & $4(44.4)$ & $0(0)$ & $0(0)$ \\
Vomiting & $2(22.2)$ & $3(33.3)$ & $3(33.3)$ & $1(11.1)$ & $0(0)$ \\
Diarrhea & $7(77.8)$ & $2(22.2)$ & $0(0)$ & $0(0)$ & $0(0)$ \\
Fatigue & $8(88.9)$ & $0(0)$ & $1(11.1)$ & $0(0)$ & $0(0)$ \\
Weight loss & $3(33.3)$ & $3(33.3)$ & $2(22.2)$ & $1(11.1)$ & $0(0)$ \\
\hline
\end{tabular}

and 1 case, respectively. The median follow-up time was 17 months. The median time of disease-free survival was 16.6 months. Four patients who received surgery and 1 patient who refused surgery showed no recurrence or metastasis at the end of the follow-up period. Of the 4 patients with distant metastasis, 2 had liver metastases, and 2 had peritoneum metastases.

\section{Discussion}

The main purpose of this study was to observe the MTD and DLT of hypo-fractionated radiotherapy for local advanced gastric cancer. The results showed that DLTs occurred at the $41.6 \mathrm{~Gy} / 2.6 \mathrm{~Gy}$ dose level; thus, we identified $40 \mathrm{~Gy} / 2.5 \mathrm{~Gy}$ as the MTD dose. The most common side effects of grade 1-3 were leucopenia, nausea, vomiting and weight loss. DLTs consisted of grade 3 vomiting and weight loss.

The MAGIC and FNCLCC/FFCD studies identified the therapeutic modalities of preoperative chemotherapy for gastric cancer $[7,8]$. The short-term response rate of radiotherapy and local regional control present obvious advantages. The phase 3 randomized controlled study from our center compared the prognosis of preoperative radiotherapy with that of surgery alone. The preoperative radiotherapy group received a 40 Gy dose of radiotherapy prior to surgery. The results showed that the 5-year and 10-year overall survival rates in the preoperative radiotherapy group were 30.1 and $19.75 \%$, respectively, which were significantly better than those in the surgery alone group (20.3 and 13.3\%, respectively; $p=0.009$ ) [9]. In the CROSS study, a similar conclusion was obtained [5]. In recent years, total neo-adjuvant treatment has become a topic of high interest in the treatment of gastric cancer. In Stahl's study, chemotherapy and radiotherapy were performed as neoadjuvant therapy, and the long-term prognosis was discussed. Three hundred and fifty-four clinical stage 
T3-4NanyM0 esophageal and gastric adenocarcinoma patients were enrolled. The control group with cisplatin plus 5-fluorouracil and leucovorin (PLF) underwent surgery, and the experimental group received radiotherapy over 3 weeks concurrent with the same chemotherapy regimen followed by surgery. No postoperative adjuvant therapy was given. Although the toxicity of the preoperative chemoradiotherapy group increased (grade 3 to 4 toxicity: $12 \%$ vs. $5 \%$ ), chemoradiotherapy significantly improved the $\mathrm{pCR}$ rate $(15.6 \%$ vs. $2 \%)$ and the pathologic N0 rate $(64.4 \%$ vs. $37.7 \%)$. The five-year overall survival rate was higher in the chemoradiotherapy group ( $39.5 \%$ vs. $24.4 \%, p=0.055)$ [3]. Although the study only recruited 126 patients due to slow recruiting speed, and the research results revealed no significant differences in overall survival, the results showed that preoperative chemoradiotherapy could improve the patient survival trend for locally advanced adenocarcinoma of the esophagogastric junction. The RTOG9904 study reported by Maurel was a single-arm phase 2 study. All the recruited patients received radiotherapy concurrent with chemotherapy (5-fluorouracil and paclitaxel) after 2 cycles of PLF chemotherapy. Radical surgery was performed 5 to 6 weeks after the neoadjuvant treatment, and no adjuvant chemotherapy was given. The study included 49 patients, and the pCR rate and R0 resection rate were 26 and 77\%, respectively. The grade 4 toxicity rate was $21 \%$ [10]. These previous clinical studies of neo-adjuvant chemoradiotherapy showed that this treatment modality could improve the pCR rate and improve the long-term outcome. Our study examined the therapeutic modalities of concurrent chemoradiotherapy and perioperative chemotherapy plus radical surgery. The pCR rate was $50 \%$ in the patients who underwent surgery. One patient received no further treatment after concurrent chemoradiotherapy, and this patient is currently in a state of disease-free survival. Therefore, the effectiveness of the total neo-adjuvant treatment modality warrants further study.

The conventional radiotherapy fraction mode is routinely used in gastric cancer. Selected studies have attempted hypo-fractionated treatment for palliative radiotherapy [11-15]. A total of 107 patients were enrolled in the study by Tey et al., in which the radiotherapy dose ranged from single 8 Gy to $40 \mathrm{~Gy} / 16 \mathrm{f}$ [15]. The entire stomach was irradiated using the AP-PA radiation technique, and the results showed that the incidence of grade 3 toxicity in the entire group was $3.8 \%$, consisting of gastrointestinal toxicities. No grade 4 or 5 toxicity occurred; therefore, the technique was considered tolerable for hypo-fractionated radiotherapy. Compared with the above studies, we used 2.5-2.7 Gy as a fraction to escalate the dose. Our target volume did not include the entire stomach, and the radiotherapy plan was performed using IMRT technology. Therefore, we believe that the fraction mode is safe and tolerable. Additionally, hypo-fractionated radiotherapy has the advantage of shortening the total neoadjuvant treatment duration and saving medical resources. Our results showed that 41.6 Gy/2.6 Gy was the DLT dose level, and the major toxicities included gastrointestinal adverse events and weight loss. No serious complications occurred during or after surgery.

In the perioperative treatment of gastric cancer, S-1 has been widely used in Asia [16, 17]. The previous phase I study from our center assessed the MTD of S-1 in postoperative chemoradiotherapy for gastric cancer [18]. The conventional fraction radiotherapy dose of $45 \mathrm{~Gy} / 1.8 \mathrm{~Gy}$ was delivered, and the MTD was $80 \mathrm{mg} / \mathrm{m}^{2}$. The same concurrent chemoradiotherapy regimen was also used in the phase II study of preoperative gastric cancer with concurrent chemoradiotherapy. Preliminary results showed that the tolerances of radiotherapy and concurrent chemotherapy reached 96.7 and $93.3 \%$, respectively [19]. However, this scheme has not been widely used in Western white populations. Several studies have reported that the level of the CYP2A6 enzyme is higher in the white population than in the Asian population, such that the maximum tolerance dose is lower [20,21]. The current study fixed the S-1 dose to $80 \mathrm{mg} / \mathrm{m}^{2}$, as recommended by the previous study, and the design was planned to escalate the hypo-fractionated radiotherapy dose.

In this study, a new modality of radiotherapy and chemotherapy for local advanced gastric cancer was discussed, thus offering a foundation for further research. However, this study observed worse prognoses than those reported in the literature, and we attempted to analyze the underlying reasons. First, the overall clinical staging of the enrolled patients was late. Seven cases were graded as stage III, and the prognosis for these patients was poor. Second, 2 patients had peritoneal metastasis shortly after chemoradiotherapy. According to past reports, the incidence of peritoneal metastasis in gastric cancer is as high as $9.8-19.3 \%$ [22, 23], and peritoneal metastasis is difficult to diagnose prior to surgery. Therefore, peritoneal metastasis is often a factor that influences staging bias and leads to poor prognosis. In addition, this study was designed for phase I clinical studies, and the sample size might have interfered with the outcome of the final prognosis. Therefore, in further large-sample studies, we intend to design additional clinical means for more accurate staging and reduced data bias.

\section{Conclusion}

In summary, hypo-fractionated radiotherapy for local advanced gastric cancer is safe and feasible and is well tolerated. The recommended dose of radiotherapy is 40 Gy/2.5 Gy. The DLTs included gastrointestinal toxicity and weight loss. Further evaluation of the response rate and long-term outcome remains to be observed in large-sample studies. 


\section{Abbreviations}

DLT: Dose-limited toxicity; MDT: Multidisciplinary team; MTD: Maximum tolerated dose; pCR: Pathological complete response; PTV: Planning target volume; TRG: Tumor regression grade

\section{Funding}

This work was supported by the National Natural Science Fund (81773241) and CAMS Innovation Fund for Medical Sciences (CIFMS)(2017-I2M-1-006). The funding body had no role in the design of the study and collection, analysis, and interpretation of data and in writing the manuscript.

\section{Availability of data and materials}

The datasets used and/or analyzed during the current study are available from the corresponding author on reasonable request.

\section{Authors' contributions}

Study design: JJi, ZD, WX and LN. Analyzed data or performed statistica analysis: LN. Drafted manuscript: LN. Reviewed and commented on the manuscript: LN, WX, TYuan, ZD, CY, YL, JL, JJu, LW, TYu, FH, LYu, SY, WS, JJi and $L Y e$. All authors read and approved the final manuscript.

\section{Ethics approval and consent to participate}

This study was approved by the ethics committee of Cancer Institute and Hospital, Chinese Academy of Medical Sciences (CH-Gl-100). All subjects signed a written informed consent form.

\section{Consent for publication}

Not applicable.

\section{Competing interests}

The authors declare that they have no competing interests.

\section{Publisher's Note}

Springer Nature remains neutral with regard to jurisdictional claims in published maps and institutional affiliations.

\section{Author details \\ 1 Department of Radiation Oncology, Chinese National Cancer Center/Cancer Hospital, Chinese Academy of Medical Sciences, 17 Panjiayuannanli, Chaoyang District, Beijing 100021, China. ${ }^{2}$ Department of Abdominal Surgical Oncology, Chinese National Cancer Center/Cancer Hospital, Chinese Academy of Medical Sciences, Beijing, China. ${ }^{3}$ Department of Medical Oncology, Chinese National Cancer Center/Cancer Hospital, Chinese Academy of Medical Sciences, Beijing, China. ${ }^{4}$ Department of Radiology, Chinese National Cancer Center/Cancer Hospital, Chinese Academy of Medical Sciences, Beijing, China.}

\section{Received: 26 February 2018 Accepted: 30 July 2018}

\section{Published online: 08 August 2018}

\section{References}

1. Chen W, Zheng R, Baade PD, Zhang S, Zeng H, Bray F, Jemal A, Yu XQ, He J. Cancer statistics in China, 2015. CA Cancer J Clin. 2016;66(2):115-32.

2. Ajani JA, Winter $\mathrm{K}$, Okawara GS, Donohue JH, Pisters PW, Crane CH, Greskovich JF, Anne PR, Bradley JD, Willett C, et al. Phase II trial of preoperative chemoradiation in patients with localized gastric adenocarcinoma (RTOG 9904): quality of combined modality therapy and pathologic response. J Clin Oncol. 2006;24(24):3953-8.

3. Stahl M, Walz MK, Riera-Knorrenschild J, Stuschke M, Sandermann A, Bitzer M, Wilke H, Budach W. Preoperative chemotherapy versus chemoradiotherapy in locally advanced adenocarcinomas of the oesophagogastric junction (POET): long-term results of a controlled randomised trial. Eur J Cancer. 2017:81:183-90.

4. Kim MS, Lim JS, Hyung WJ, Lee YC, Rha SY, Keum KC, Koom WS Neoadjuvant chemoradiotherapy followed by D2 gastrectomy in locally advanced gastric cancer. World J Gastroenterol. 2015;21(9):2711-8.

5. Shapiro J, van Lanschot JJ, Hulshof MC, van Hagen P, van Berge Henegouwen MI, Wijnhoven BP, van Laarhoven HW, Nieuwenhuijzen GA, Hospers GA, Bonenkamp JJ, et al. Neoadjuvant chemoradiotherapy plus surgery versus surgery alone for oesophageal or junctional cancer (CROSS): long-term results of a randomised controlled trial. Lancet Oncol. 2015;16(9):1090-8.
6. Mandard AM, Dalibard F, Mandard JC, Marnay J, Henry-Amar M, Petiot JF, Roussel A, Jacob JH, Segol P, Samama G, et al. Pathologic assessment of tumor regression after preoperative chemoradiotherapy of esophageal carcinoma. Clinicopathologic correlations. Cancer. 1994;73(11):2680-6.

7. Cunningham D, Allum WH, Stenning SP, Thompson JN, Van de Velde CJ, Nicolson M, Scarffe JH, Lofts FJ, Falk SJ, Iveson TJ, et al. Perioperative chemotherapy versus surgery alone for resectable gastroesophageal cancer. N Engl J Med. 2006;355(1):11-20.

8. Ychou M, Boige V, Pignon JP, Conroy T, Bouche O, Lebreton G, Ducourtieux M, Bedenne L, Fabre JM, Saint-Aubert B, et al. Perioperative chemotherapy compared with surgery alone for resectable gastroesophageal adenocarcinoma: an FNCLCC and FFCD multicenter phase III trial. J Clin Oncol. 2011;29(13):1715-21.

9. Zhang ZX, Gu XZ, Yin WB, Huang GJ, Zhang DW, Zhang RG. Randomized clinical trial on the combination of preoperative irradiation and surgery in the treatment of adenocarcinoma of gastric cardia (AGC)--report on 370 patients. Int J Radiat Oncol Biol Phys. 1998;42(5):929-34.

10. Pera M, Gallego R, Montagut C, Martin-Richard M, Iglesias M, Conill C, Reig A, Balague C, Petriz L, Momblan D, et al. Phase II trial of preoperative chemoradiotherapy with oxaliplatin, cisplatin, and 5-FU in locally advanced esophageal and gastric cancer. Ann Oncol. 2012;23(3):664-70.

11. Kim MM, Rana V, Janjan NA, Das P, Phan AT, Delclos ME, Mansfield PF, Ajani JA, Crane $\mathrm{CH}$, Krishnan S. Clinical benefit of palliative radiation therapy in advanced gastric cancer. Acta Oncol. 2008;47(3):421-7.

12. Hashimoto K, Mayahara H, Takashima A, Nakajima TE, Kato K, Hamaguchi T, Ito $Y$, Yamada Y, Kagami Y, Itami J, et al. Palliative radiation therapy for hemorrhage of unresectable gastric cancer: a single institute experience. J Cancer Res Clin Oncol. 2009;135(8):1117-23.

13. Asakura H, Hashimoto T, Harada H, Mizumoto M, Furutani K, Hasuike N, Matsuoka M, Ono H, Boku N, Nishimura T. Palliative radiotherapy for bleeding from advanced gastric cancer: is a schedule of $30 \mathrm{~Gy}$ in 10 fractions adequate? J Cancer Res Clin Oncol. 2011;137(1):125-30.

14. Chaw CL, Niblock PG, Chaw CS, Adamson DJ. The role of palliative radiotherapy for haemostasis in unresectable gastric cancer: a singleinstitution experience. Ecancermedicalscience. 2014;8:384.

15. Tey J, Choo BA, Leong CN, Loy EY, Wong LC, Lim K, Lu JJ, Koh WY. Clinical outcome of palliative radiotherapy for locally advanced symptomatic gastric cancer in the modern era. Medicine (Baltimore). 2014;93(22):e118.

16. Bang YJ, Kim YW, Yang HK, Chung HC, Park YK, Lee KH, Lee KW, Kim YH, Noh SI, Cho JY, et al. Adjuvant capecitabine and oxaliplatin for gastric cancer after D2 gastrectomy (CLASSIC): a phase 3 open-label, randomised controlled trial. Lancet. 2012;379(9813):315-21.

17. Sakuramoto S, Sasako M, Yamaguchi T, Kinoshita T, Fujii M, Nashimoto A, Furukawa $\mathrm{H}$, Nakajima T, Ohashi Y, Imamura $\mathrm{H}$, et al. Adjuvant chemotherapy for gastric cancer with S-1, an oral fluoropyrimidine. N Engl J Med. 2007;357(18):1810-20.

18. Wang $X$, Zhao DB, Yang L, Chi Y, Tang Y, Li N, Wang SL, Song YW, Liu YP, Liu WY et al. S-1 chemotherapy and intensity-modulated radiotherapy after D1/D2 lymph node dissection in patients with node-positive gastric cancer: a phase I/II study. Br J Cancer. 2018;118(3):338-43.

19. Wang X, Zhao DB, Jin J, Chi Y, Yang L, Tang Y, Che X, Zhao H, Jiang LM, Ren H. A randomized phase II trial of neoadjuvant chemotherapy compared with Chemoradiation therapy in locally advanced gastroesophageal and gastric adenocarcinoma: preliminary results. Int J Radiat Oncol. 2016;96(2S):S32.

20. Ajani JA, Faust J, Ikeda K, Yao JC, Anbe H, Carr KL, Houghton M, Urrea P. Phase I pharmacokinetic study of S-1 plus cisplatin in patients with advanced gastric carcinoma. J Clin Oncol. 2005;23(28):6957-65.

21. Ajani JA, Rodriguez W, Bodoky G, Moiseyenko V, Lichinitser M, Gorbunova V, Vynnychenko I, Garin A, Lang I, Falcon S. Multicenter phase III comparison of cisplatin/S-1 with cisplatin/infusional fluorouracil in advanced gastric or gastroesophageal adenocarcinoma study: the FLAGS trial. J Clin Oncol. 2010;28(9):1547-53.

22. Spolverato G, Ejaz A, Kim Y, Squires MH, Poultsides GA, Fields RC, Schmidt C, Weber SM, Votanopoulos K, Maithel SK, et al. Rates and patterns of recurrence after curative intent resection for gastric cancer: a United States multi-institutional analysis. J Am Coll Surg. 2014;219(4):664-75.

23. Wang $X$, Jin J, Li Y, Wang W, Liu Y, Song Y, Liu X, Yu Z. Prognostic factor for locally advanced gastric or gastroesophageal cancer patients after curative gastrectomy and indications for adjuvant therapy. Chin J Radiat Oncol. 2011;20(4):306-11. 